\title{
On the estimation of spread rate for a biological population
}

\author{
Jim Clark ${ }^{\mathrm{a}, 1}$, Lajos Horváth ${ }^{\mathrm{b}, *}$, Mark Lewis ${ }^{\mathrm{b}, 2}$ \\ ${ }^{a}$ Department of Botany and Earth \& Ocean Sciences, Duke University, Durham, NC 27708, USA \\ ${ }^{\mathrm{b}}$ Department of Mathematics, University of Utah, 155 South 1440 East, Salt Lake City, UT 84112-0090, USA
}

Received August 1999; received in revised form February 2000

\begin{abstract}
We propose a nonparametric estimator for the rate of spread of an introduced population. We prove that the limit distribution of the estimator is normal or stable, depending on the behavior of the moment generating function. We show that resampling methods can also be used to approximate the distribution of the estimators. (C) 2001 Elsevier Science B.V. All rights reserved
\end{abstract}

$M S C$ : primary $62 \mathrm{G} 05$; secondary $62 \mathrm{G} 20$

Keywords: Moment-generating function; Normal distribution; Bootstrap

\section{Introduction and results}

Ecologists use dispersal kernels to estimate the speed at which an introduced population might invade new environments. A kernel is fitted to the scatter of offspring locations about a parent, and this kernel is then used to calculate a velocity of spread. There is growing awareness that these estimates can be extremely sensitive to assumptions about kernel shape (Kot et al., 1996; Clark, 1998); differences in model forms that appear subtle (and fit data sets equally well), may imply large differences in velocity estimates.

We demonstrate a method that sidesteps entirely assumptions concerning kernel shape by advancing directly from empirical dispersal data to an estimator for spread rate.

A classical model for biological invasions is the integrodifference equation

$$
u_{s+1}(x)=\int_{-\infty}^{\infty} R_{0} G\left(u_{s}(x-y)\right) \mathrm{d} F(y), \quad s=0,1, \ldots,
$$

\footnotetext{
* Corresponding author. Tel.: +1-801-581-8159; fax: +1-801-581-4148.

E-mail address: horvath@math.utah.edu (L. Horváth).

${ }^{1}$ Research partially supported by National Science Foundation grants DEB-9632854 and DEB-9453498.

${ }^{2}$ Research partially supported by National Science Foundation grants DMS-9457816, DMS-9973212 and by the Institute for Mathematics and its Applications with funds provided by the National Science Foundation.
} 
where $u_{s}(x)$ is the density of invading organisms at location $x \in R$ and time $s, R_{0}>1$ is the geometric growth rate of the population, $G(u)$ describes nonlinear growth dynamics, and $F$ is the distribution function of a random variable $X$ describing the distance an individual disperses in one time step. Here it is assumed that $R_{0} G(u)$ has fixed points at $u=0$ and $u=1, R_{0} G(u)>u$ for $0<u<1, G^{\prime}(0)=1$ and $\sup _{0<u \leqslant 1} G(u) / u=1$. Thus, the maximum per capita geometric growth is $R_{0}$ which occurs as the population density approaches 0 (Weinberger, 1982). The asymptotic spread rate of the solutions arising from compact initial data can be calculated under the assumption that the moment generating function of $X$

$$
M(t)=\int_{-\infty}^{\infty} \mathrm{e}^{t x} \mathrm{~d} F(x)
$$

exists on some nonzero interval $\left[0, t_{0}\right)$. Weinberger (1982) showed that under a wide variety of assumptions on reproduction and dispersal, the rate of spread of a locally introduced population asymptotically approaches

$$
c_{0}=\inf _{s>0} Z(s)
$$

as the time since the initial release becomes large. Here

$$
Z(t)=\frac{1}{t} \log \left(R_{0} M(t)\right), \quad 0<t<t_{0} .
$$

The function $Z(t)$ is continuous and can be shown to have a unique critical point $\tau$ which gives a global minimum for $Z(t)$ and thus $Z^{\prime}(\tau)=0$ and

$$
c_{0}=Z(\tau) .
$$

This was proved by Weinberger (1978) for density functions $f=F^{\prime}$ with bounded support (see Lemma 4.1 in Weinberger (1978) and its proof) and a straightforward extension of the proof includes the general case above (cf. Lemma 9.1 in Weinberger, 1982).

Biological measurements of dispersal distances may be available without the knowledge of the underlying distribution function. We consider how to estimate $c_{0}$ in this case. We assume that the observations $X_{1}, X_{2}, \ldots, X_{n}$ are independent, identically distributed random variables with distribution function $F$. Since we cannot assume any parametric form for $F$ (cf. the empirical example in Kot et al. (1996)) we use a nonparametric approach. We consider the estimation of $M(t)$ with the empirical moment generating function

$$
M_{n}(t)=\frac{1}{n} \sum_{1 \leqslant i \leqslant n} \exp \left(t X_{i}\right), \quad 0 \leqslant t<\infty .
$$

This suggests that

$$
Z_{n}(t)=\frac{1}{t} \log \left(R_{0} M_{n}(t)\right), \quad 0<t<\infty
$$

can be used as a nonparametric estimator for $Z(t)$ and thus,

$$
\hat{c}_{n}=Z_{n}\left(\hat{\tau}_{n}\right)
$$

can be used as a nonparametric estimator for $c_{0}$, where

$$
Z_{n}^{\prime}\left(\hat{\tau}_{n}\right)=0
$$

We wish to note that the estimation of $\tau$ and $c_{0}$ fits into the general scheme of estimation based on Laplace transforms. Csörgö and Teugels (1990) introduced and investigated estimation of parameters using empirical Laplace transforms and used the general scheme in five different scenarios.

Let $t_{0}=\sup \{t>0: M(t)<\infty\} \leqslant \infty$ and assume throughout that $t_{0}>0$. Our first result is the strong consistency of $\hat{\tau}_{n}$ and $\hat{c}_{n}$. 
Theorem 1.1. If

$$
\tau<t_{0}
$$

and

$$
Z^{\prime \prime}(\tau) \neq 0
$$

then we have

$$
\hat{\tau}_{n} \rightarrow \tau \quad \text { a.s. }
$$

If (1.1) also holds, then

$$
\hat{c}_{n} \rightarrow c_{0} \quad \text { a.s. }
$$

The proof of Theorem 1.1 will be given in Section 3. Next, we consider the asymptotic distributions of $\hat{c}_{n}-c_{0}$ and $\hat{\tau}_{n}-\tau$. Let $N(a, b)$ be a normal random variable with mean $a$ and variance $b \geqslant 0$,

$$
c_{1}(t)=\frac{1}{t M(t)}
$$

and

$$
c_{2}(t)=-\left\{\frac{1}{t^{2} M(t)}+\frac{M^{\prime}(t)}{t M^{2}(t)}\right\} .
$$

Theorem 1.2. If

$$
\tau<t_{0} / 2
$$

and (1.3) holds, then

$$
n^{1 / 2}\left(\hat{\tau}_{n}-\tau\right) \stackrel{\mathscr{D}}{\rightarrow} N\left(0, \sigma^{2}\right)
$$

with

$$
\begin{aligned}
\sigma^{2}= & \left(Z^{\prime \prime}(\tau)\right)^{-2}\left\{c_{1}^{2}(\tau)\left(M^{\prime \prime}(2 \tau)-\left(M^{\prime}(\tau)\right)^{2}\right)+2 c_{1}(\tau) c_{2}(\tau)\left(M^{\prime}(2 \tau)\right.\right. \\
& \left.\left.-M(\tau) M^{\prime}(\tau)\right)+c_{2}^{2}(\tau)\left(M(2 \tau)-M^{2}(\tau)\right)\right\} .
\end{aligned}
$$

If (1.1) also holds, then

$$
n^{1 / 2}\left(\hat{c}_{n}-c_{0}\right) \stackrel{\mathscr{D}}{\rightarrow} N\left(0, v^{2}\right)
$$

with

$$
\begin{aligned}
v^{2}= & c_{1}^{2}(\tau)\left(M(2 \tau)-M^{2}(\tau)\right)-2\left\{Z^{\prime}(\tau) / Z^{\prime \prime}(\tau)\right\}\left\{c _ { 1 } ^ { 2 } ( \tau ) \left(M^{\prime}(2 \tau)\right.\right. \\
& \left.\left.-M(\tau) M^{\prime}(\tau)\right)+c_{1}(\tau) c_{2}(\tau)\left(M(2 \tau)-M^{2}(\tau)\right)\right\} \\
& +\left\{Z^{\prime}(\tau) / Z^{\prime \prime}(\tau)\right\}^{2}\left\{c_{1}^{2}(\tau)\left(M^{\prime \prime}(2 \tau)-\left(M^{\prime}(\tau)\right)^{2}\right)+2 c_{1}(\tau) c_{2}(\tau)\left(M^{\prime}(2 \tau)\right.\right. \\
& \left.\left.-M(\tau) M^{\prime}(\tau)\right)+c_{2}^{2}(\tau)\left(M(2 \tau)-M^{2}(\tau)\right)\right\}
\end{aligned}
$$

Condition (1.6) essentially means that $\exp (\tau X)$ has more than two moments. Condition (1.6) may be violated in some important cases. For example, if $X$ is an exponential random variable and $R_{0}$ is large, then 
(1.6) will be false. In the next theorem we consider the case when $t_{0} / 2<\tau<t_{0}$. Let $\xi_{\alpha}, 1<\alpha<2$ be a stable random variable with index $\alpha$. We say that the random variable $Y$ is the domain of attraction of $\xi_{\alpha}$, if

$$
\sum_{1 \leqslant i \leqslant n}\left(Y_{i}-E Y_{i}\right) /\left(n^{1 / \alpha} L(n)\right) \stackrel{\mathscr{D}}{\rightarrow} \xi_{\alpha}
$$

with some slowly varying function $L$, where $Y_{1}, Y_{2}, \ldots, Y_{n}$ are independent copies of $Y$. For the properties of slowly varying functions we refer to Bingham et al. (1987).

Theorem 1.3. If (1.3) holds and $X \exp (\tau X)$ is in the domain of attraction of $\xi_{\alpha}$ with some $1<\alpha<2$, then there is a slowly varying function $L_{1}(n)$ such that

$$
n^{1-1 / \alpha} L_{1}(n)\left(\hat{\tau}_{n}-\tau\right) \stackrel{\mathscr{D}}{\rightarrow} \xi_{\alpha} .
$$

If (1.1) also holds, then there is a slowly varying function $L_{2}(n)$ such that

$$
n^{1-1 / \alpha} L_{2}(n)\left(\hat{c}_{n}-c_{0}\right) \stackrel{\mathscr{D}}{\rightarrow} \xi_{\alpha} .
$$

We note that if $X \exp (\tau X)$ is in the domain of attraction of $\xi_{\alpha}$, then $M(\tau \alpha-\varepsilon)<\infty$ and $M(\tau \alpha+\varepsilon)=\infty$ for all $0<\varepsilon<\tau \alpha$.

\section{Bootstrap}

If we wish to use (1.8) to construct confidence intervals or for hypothesis testing we need the value of the asymptotic variance $v^{2}$. Since $v^{2}$ is unknown we must estimate it from the random sample. If $v_{n}$ satisfies

$$
\left|v_{n}-v\right|=\mathrm{oP}_{\mathrm{P}}(1)
$$

then under the conditions of Theorem 1.2 we have that

$$
\frac{n^{1 / 2}\left(\hat{c}_{n}-c_{0}\right)}{v_{n}} \stackrel{\mathscr{D}}{\rightarrow} N(0,1) .
$$

In the proof of (1.8) we show that

$$
n^{1 / 2}\left(\hat{c}_{n}-c_{0}\right)=b_{0}(\tau) n^{1 / 2}\left(M_{n}(\tau)-M(\tau)\right)+b_{1}(\tau) n^{1 / 2}\left(M_{n}^{\prime}(\tau)-M^{\prime}(\tau)\right)+\mathrm{o}_{\mathrm{P}}(1),
$$

where $b_{0}(\tau)$ and $b_{1}(\tau)$ are easily computable functions of $M(\tau), M^{\prime}(\tau)$ and $M^{\prime \prime}(\tau)$, say $b_{0}(\tau)=b_{0}\left(M(\tau), M^{\prime}(\tau)\right.$, $\left.M^{\prime \prime}(\tau)\right)$ and $b_{1}(\tau)=b_{1}\left(M(\tau), M^{\prime}(\tau), M^{\prime \prime}(\tau)\right)$. Hence,

$$
\begin{aligned}
v^{2}= & b_{0}^{2}(\tau)\left(M(2 \tau)-M^{2}(\tau)\right)+b_{1}^{2}(\tau)\left(E X^{2} \exp (2 \tau X)-\left(M^{\prime}(\tau)\right)^{2}\right) \\
& +2 b_{0}(\tau) b_{1}(\tau)\left\{E X \exp (2 \tau X)-M(\tau) M^{\prime}(\tau)\right\}
\end{aligned}
$$

and therefore the "plug in" method (i.e. replacing all expected values by the corresponding averages) gives

$$
\begin{aligned}
v_{n}^{2}= & \hat{b}_{0}^{2}\left\{M_{n}\left(2 \hat{\tau}_{n}\right)-M_{n}^{2}\left(\hat{\tau}_{n}\right)\right\}+\hat{b}_{1}^{2}\left\{\frac{1}{n} \sum_{1 \leqslant i \leqslant n} X_{i}^{2} \exp \left(2 \hat{\tau}_{n} X_{i}\right)-\left(M_{n}^{\prime}\left(\hat{\tau}_{n}\right)\right)^{2}\right\} \\
& +2 \hat{b}_{0} \hat{b}_{1}\left\{\frac{1}{n} \sum_{1 \leqslant i \leqslant n} X_{i} \exp \left(2 \hat{\tau}_{n} X_{i}\right)-M_{n}\left(\hat{\tau}_{n}\right) M_{n}^{\prime}\left(\hat{\tau}_{n}\right)\right\},
\end{aligned}
$$

where $\hat{b}_{0}=b_{0}\left(M_{n}\left(\hat{\tau}_{n}\right), M_{n}^{\prime}\left(\hat{\tau}_{n}\right), M_{n}^{\prime \prime}\left(\hat{\tau}_{n}\right)\right)$ and $\hat{b}_{1}=b_{1}\left(M_{n}\left(\hat{\tau}_{n}\right), M_{n}^{\prime}\left(\hat{\tau}_{n}\right), M_{n}^{\prime \prime}\left(\hat{\tau}_{n}\right)\right)$. It is easy to see that $v_{n}$ of $(2.3)$ satisfies (2.1). 
Usually, the resampling methods provide better estimates for $v^{2}$ than the "plug in" method. For example, the jackknife can be used to get estimators for $v^{2}$ which satisfy (2.1). For properties and implementations of jackknife estimators for variance we refer to Shao and Tu (1995).

In this paper, we suggest the application of the "naive" bootstrap to estimate the distribution function of $n^{1 / 2}\left(\hat{c}_{n}-c_{0}\right)$. Other versions of the bootstrap resampling can also be used with minor modifications of our procedure. Following Efron (1979), let $X_{1}^{*}, X_{2}^{*}, \ldots, X_{m}^{*}$ be a random sample with distribution function

$$
F_{n}(t)=\frac{1}{n} \sum_{1 \leqslant i \leqslant n} I\left\{X_{i} \leqslant t\right\} .
$$

This means, that conditionally on $\boldsymbol{X}_{n}=\left(X_{1}, \ldots, X_{n}\right), X_{1}^{*}, X_{2}^{*}, \ldots, X_{m}^{*}$ are independent, identically distributed random variables with distribution function $F_{n}(t)$. Using the bootstrap sample $X_{1}^{*}, X_{2}^{*}, \ldots, X_{m}^{*}$ we compute the bootstrapped version of $M_{n}(t)$ and $Z_{n}(t)$ defined as

$$
M_{m, n}(t)=\frac{1}{m} \sum_{1 \leqslant i \leqslant m} \exp \left(t X_{i}^{*}\right)
$$

and

$$
Z_{m, n}(t)=\frac{1}{t} \log \left(R_{0} M_{m, n}(t)\right)
$$

Let

$$
\hat{\tau}_{m, n}=\inf \left\{t>0: Z_{m, n}^{\prime}(t)=0\right\}
$$

and

$$
\hat{c}_{m, n}=Z_{m, n}\left(\hat{\tau}_{m, n}\right)
$$

denote the bootstrap estimates for $\tau$ and $c_{0}$. Our result shows that the bootstrap can be used to simulate the distribution function of $n^{1 / 2}\left(\hat{c}_{n}-c_{0}\right)$.

Theorem 2.1. If the conditions of Theorem 1.2 are satisfied, then

$$
\sup _{-\infty<x<\infty}\left|P\left\{m^{1 / 2}\left(\hat{\tau}_{m, n}-\hat{\tau}_{n}\right) \leqslant x \mid \boldsymbol{X}_{n}\right\}-P\left\{n^{1 / 2}\left(\hat{\tau}_{n}-\tau\right) \leqslant x\right\}\right| \rightarrow 0 \quad \text { a.s. }
$$

and

$$
\sup _{-\infty<x<\infty}\left|P\left\{m^{1 / 2}\left(\hat{c}_{m, n}-\hat{c}_{n}\right) \leqslant x \mid \boldsymbol{X}_{n}\right\}-P\left\{n^{1 / 2}\left(\hat{c}_{n}-c_{0}\right) \leqslant x\right\}\right| \rightarrow 0 \quad \text { a.s. }
$$

as $\min (m, n) \rightarrow \infty$.

By repeated Monte Carlo simulations we can produce as many copies of $m^{1 / 2}\left(\hat{c}_{m, n}-\hat{c}_{n}\right)$ as we wish, the empirical distribution function of the copies of $m^{1 / 2}\left(\hat{c}_{m, n}-\hat{c}_{n}\right)$ can be used as an estimator for $P\left\{n^{1 / 2}\left(\hat{c}_{n}-c_{0}\right) \leqslant x\right\}$.

The proof of Theorem 2.1 is outlined at the end of Section 3.

\section{Proofs}

The proof of Theorem 1.1 will be based on the following lemma. 
Lemma 3.1. For any $0<T<t_{0}$ we have

$$
\begin{aligned}
& \sup _{0 \leqslant t \leqslant T}\left|M_{n}(t)-M(t)\right| \rightarrow 0 \quad \text { a.s. } \\
& \sup _{0 \leqslant t \leqslant T}\left|M_{n}^{\prime}(t)-M^{\prime}(t)\right| \rightarrow 0 \quad \text { a.s. } \\
& \sup _{0 \leqslant t \leqslant T}\left|M_{n}^{\prime \prime}(t)-M^{\prime \prime}(t)\right| \rightarrow 0 \quad \text { a.s. }
\end{aligned}
$$

Proof. Let

$$
\begin{aligned}
& M_{n}^{(1)}(t)=\frac{1}{n} \sum_{1 \leqslant i \leqslant n} \exp \left(t X_{i}\right) I\left\{X_{i} \geqslant 0\right\} \\
& M_{n}^{(2)}(t)=\frac{1}{n} \sum_{1 \leqslant i \leqslant n} \exp \left(t X_{i}\right) I\left\{X_{i}<0\right\} \\
& M^{(1)}(t)=E M_{n}^{(1)}(t)=\int_{0}^{\infty} \mathrm{e}^{t x} \mathrm{~d} F(x)
\end{aligned}
$$

and

$$
M^{(2)}(t)=E M_{n}^{(2)}(t)=\int_{-\infty}^{0} \mathrm{e}^{t x} \mathrm{~d} F(x) .
$$

Proposition 1 of Csörgő and Teugels (1990) (cf. also Csörgő, 1980) yields that

$$
\sup _{0 \leqslant t \leqslant T}\left|M_{n}^{(1)}(t)-M^{(1)}(t)\right| \rightarrow 0 \quad \text { a.s. }
$$

and

$$
\sup _{0 \leqslant t \leqslant T}\left|M_{n}^{(2)}(t)-M^{(2)}(t)\right| \rightarrow 0 \quad \text { a.s. }
$$

which give (3.1). Similarly to (3.4), Proposition 1 of Csörgő and Teugels (1990) gives the strong uniform convergence of all derivatives of $M_{n}^{(1)}(t)$ and $M_{n}^{(2)}(t)$ and, therefore, (3.2) and (3.3) are also proven.

Proof of Theorem 1.1. We have that

$$
Z_{n}^{\prime}(t)=-\frac{\log \left(R_{0} M_{n}(t)\right)}{t^{2}}+\frac{M_{n}^{\prime}(t)}{t M_{n}(t)} .
$$

Since

$$
\lim _{t \downarrow 0} M_{n}(t)=1 \quad \text { and } \quad \lim _{t \downarrow 0} M_{n}^{\prime}(t)=\frac{1}{n} \sum_{1 \leqslant i \leqslant n} X_{i},
$$

we get that

$$
\lim _{t \downarrow 0} Z_{n}^{\prime}(t)=-\infty .
$$

By Lemma 3.1 we have that

$$
\sup _{0 \leqslant t \leqslant \varepsilon} \frac{M_{n}^{\prime}(t)}{M_{n}(t)} \rightarrow \sup _{0 \leqslant t \leqslant \varepsilon} \frac{M^{\prime}(t)}{M(t)} \text { a.s. }
$$


for any $0<\varepsilon<t_{0}$ and

$$
\lim _{\varepsilon \downarrow 0} \sup _{0 \leqslant t \leqslant \varepsilon} \frac{M^{\prime}(t)}{M(t)}=E X .
$$

Let $0<\varepsilon<\min \left\{\left(\log R_{0}\right) /(4 E X+4), t_{0}\right\}$. Putting together (3.5) and (3.6) we get that there is a random variable $n_{1}=n_{1}(\omega)$ such that

$$
\sup _{0 \leqslant t \leqslant \varepsilon} \frac{M_{n}^{\prime}(t)}{M_{n}(t)} \leqslant E X+1 \quad \text { if } n \geqslant n_{1}
$$

However,

$$
\inf _{0 \leqslant t \leqslant \varepsilon} \frac{1}{t} \log \left(R_{0} M_{n}(t)\right) \geqslant \frac{1}{\varepsilon} \inf _{0 \leqslant t \leqslant \varepsilon} \log \left(R_{0} M_{n}(t)\right)
$$

and by Lemma 3.1 there is a random variable $n_{2}=n_{2}(\omega)$ such that

$$
\inf _{0 \leqslant t \leqslant \varepsilon} \log \left(R_{0} M_{n}(t)\right) \geqslant \frac{1}{2} \log R_{0} \quad \text { if } n \geqslant n_{2}(\omega)
$$

If $n \geqslant \max \left(n_{1}, n_{2}\right)$ we have that

$$
\begin{aligned}
\sup _{0 \leqslant t \leqslant \varepsilon} t Z_{n}^{\prime}(t) & \leqslant \sup _{0 \leqslant t \leqslant \varepsilon} \frac{M_{n}^{\prime}(t)}{M_{n}(t)}+\sup _{0 \leqslant t \leqslant \varepsilon} \frac{-\log \left(R_{0} M_{n}(t)\right)}{t} \\
& \leqslant E X+1-\frac{1}{2 \varepsilon} \log R_{0} \\
& \leqslant-\frac{1}{\varepsilon} \frac{\log R_{0}}{4} .
\end{aligned}
$$

This means that there is a random variable $n_{0}=n_{0}(\omega) \geqslant \max \left(n_{1}, n_{2}\right)$ and $\varepsilon>0$ such that $Z_{n}^{\prime}(t)<-1$ for all $0 \leqslant t \leqslant \varepsilon$, if $n \geqslant n_{0}$.

Since $Z^{\prime}(t) \rightarrow-\infty$ as $t \downarrow 0$ we have local minimum at $\tau$. Theorem $A$ in Hardy (1996, p. 232) yields that for any $\delta^{*}$ there are $\delta>0$ and $\varepsilon \leqslant \tau-\delta^{*} \leqslant \eta_{1}<\tau<\eta_{2} \leqslant \tau+\delta^{*}<t_{0}$ such that $Z^{\prime}\left(\eta_{1}\right)<-\delta$ and $Z^{\prime}\left(\eta_{2}\right)>\delta$. Let $\eta_{2}<T<t_{0}$. By Lemma 3.1 we have that

$$
\sup _{\varepsilon \leqslant t \leqslant T}\left|Z_{n}^{\prime}(t)-Z^{\prime}(t)\right| \rightarrow 0 \quad \text { a.s. }
$$

and therefore $Z_{n}^{\prime}\left(\eta_{1}\right) \leqslant-\delta / 2$ and $Z_{n}^{\prime}\left(\eta_{2}\right) \geqslant \delta / 2$, if $n \geqslant n_{3}=n_{3}(\omega)$. Since $Z_{n}^{\prime}(t)$ is continuous on $\left[\eta_{1}, \eta_{2}\right]$, there is $\tau_{n}^{*} \in\left(\eta_{1}, \eta_{2}\right)$ such that $A_{n}^{\prime}\left(\tau_{n}^{*}\right)=0$. Thus, we showed that there is a sequence $\tau_{n}^{*}$ such that $Z_{n}^{\prime}\left(\tau_{n}^{*}\right)=0$ and $\tau_{n}^{*} \rightarrow \tau$ a.s. If there is a subsequence $\varepsilon \leqslant \gamma_{n(k)}$ satisfying $Z_{n}^{\prime}\left(\gamma_{n(k)}\right)=0$ and $\gamma_{n(k)} \rightarrow \gamma<\tau$ a.s., then (3.7) implies that $Z^{\prime}(\gamma)=0$ contradicting the definition of $\tau$. Hence (1.4) is proven.

Similarly to (3.7) one can show that

$$
\sup _{\varepsilon \leqslant t \leqslant T}\left|Z_{n}(t)-Z(t)\right| \rightarrow 0 \quad \text { a.s. }
$$

for any $0<\varepsilon<T<t_{0}$. The continuity of $Z(t)$ and (1.4) gives that

$$
Z\left(\tau_{n}\right) \rightarrow Z(\tau)=c_{0}
$$

and therefore (1.5) follows from (1.4).

The next lemma will be used in the proofs of Theorems 1.2 and 1.3. 
Lemma 3.2. We have that

$$
\left|Z_{n}(t)-Z(t)-\frac{1}{n} \sum_{1 \leqslant i \leqslant n} \xi_{i}(t)\right|=\mathrm{O}_{\mathrm{P}}(1)\left(M_{n}(t)-M(t)\right)^{2}
$$

and

$$
\left|Z_{n}^{\prime}(t)-Z^{\prime}(t)-\frac{1}{n} \sum_{1 \leqslant i \leqslant n} \eta_{i}(t)\right|=\mathrm{O}_{\mathrm{P}}(1)\left(M_{n}(t)-M(t)\right)^{2}+\mathrm{O}_{\mathrm{P}}(1)\left(M_{n}^{\prime}(t)-M^{\prime}(t)\right)^{2}
$$

for any $0<t<t_{0}$, where

$$
\xi_{i}(t)=c_{1}(t)\left(\exp \left(t X_{i}\right)-M(t)\right)
$$

and

$$
\eta_{i}(t)=c_{1}(t)\left(X_{i} \exp \left(t X_{i}\right)-M^{\prime}(t)\right)+c_{2}(t)\left(\exp \left(t X_{i}\right)-M(t)\right) .
$$

Proof. The mean-value theorem gives

$$
\begin{aligned}
Z_{n}(t)-Z(t) & =\frac{1}{t}\left\{\log M_{n}(t)-\log M(t)\right\} \\
& =\frac{1}{t M(t)}\left(M_{n}(t)-M(t)\right)-\frac{1}{t \mu^{2}}\left(M_{n}(t)-M(t)\right)^{2},
\end{aligned}
$$

where $\mu$ is a point between $M_{n}(t)$ and $M(t)$. Now (3.9) follows from Lemma 3.1 with the choice of $c(t)=$ $1 /(t M(t))$.

Similar arguments give (3.10). The details are omitted.

Lemma 3.3. If (1.1) holds, then

$$
\left|\hat{\tau}_{n}-\tau-\left(Z^{\prime}(\tau)-Z_{n}^{\prime}(\tau)\right) / Z^{\prime \prime}(\tau)\right|=\mathrm{oP}_{\mathrm{P}}(1)\left|Z_{n}^{\prime}(\tau)-Z^{\prime}(\tau)\right|
$$

and

$$
\left|\hat{c}_{n}-c-\left\{Z_{n}(\tau)-Z(\tau)-Z^{\prime}(\tau)\left(Z_{n}^{\prime}(\tau)-Z^{\prime}(\tau)\right) / Z^{\prime \prime}(\tau)\right\}\right|=\mathrm{op}_{\mathrm{P}}(1)\left|Z_{n}^{\prime}(\tau)-Z^{\prime}(\tau)\right| .
$$

Proof. Similarly to (3.7) we have

$$
\sup _{\varepsilon \leqslant t \leqslant T}\left|Z_{n}^{\prime \prime}(t)-Z^{\prime \prime}(t)\right| \rightarrow 0 \quad \text { a.s. }
$$

for any $0<\varepsilon<T<t_{0} / 2$. The mean value theorem gives

$$
Z_{n}^{\prime}\left(\hat{\tau}_{n}\right)-Z_{n}^{\prime}(\tau)=Z_{n}^{\prime \prime}(\xi)\left(\hat{\tau}_{n}-\tau\right),
$$

where $\xi$ is between $\hat{\tau}_{n}$ and $\tau$. By definition $Z_{n}^{\prime}\left(\hat{\tau}_{n}\right)=0, Z^{\prime}(\tau)=0$ and, therefore,

$$
\hat{\tau}_{n}-\tau=-\frac{Z_{n}^{\prime}(\tau)}{Z_{n}^{\prime \prime}(\xi)}=-\frac{Z_{n}^{\prime}(\tau)-Z^{\prime}(\tau)}{Z_{n}^{\prime \prime}(\xi)} .
$$

Hence, (3.11) follows from (3.13).

Next, we write

$$
\begin{aligned}
\hat{c}_{n}-c_{0} & =Z_{n}\left(\hat{\tau}_{n}\right)-Z(\tau) \\
& =Z_{n}\left(\hat{\tau}_{n}\right)-Z_{n}(\tau)+Z_{n}(\tau)-Z(\tau) \\
& =Z_{n}^{\prime}(\xi)\left(\hat{\tau}_{n}-\tau\right)+Z_{n}(\tau)-Z(\tau),
\end{aligned}
$$

where $\xi$ is between $\hat{\tau}_{n}$ and $\tau$. Using (3.7) and (3.11) we obtain immediately (3.12). 
Proof of Theorem 1.2. According to Lemmas 3.2 and 3.3, the proof of (1.7) is complete if we show that

$$
\frac{n^{-1 / 2}}{Z^{\prime \prime}(\tau)} \sum_{1 \leqslant i \leqslant n} \xi_{i}(\tau) \stackrel{\mathscr{P}}{\rightarrow} N\left(0, \sigma^{2}\right)
$$

Condition (1.6) implies that $E \xi_{i}^{2}(\tau)<\infty$ and, therefore, (3.14) is an immediate consequence of the central limit theorem.

Observing that $E \eta_{i}^{2}(t)<\infty$ and $E \xi_{i}^{2}(t)<\infty$, by the central limit theorem we have

$$
n^{-1 / 2}\left(\sum_{1 \leqslant i \leqslant n} \xi_{i}(\tau)-Z^{\prime}(\tau) \sum_{1 \leqslant i \leqslant n} \eta_{i}(\tau) / Z^{\prime \prime}(\tau)\right) \stackrel{\mathscr{D}}{\rightarrow} N\left(0, v^{2}\right)
$$

and, therefore, (1.8) follows from Lemmas 3.2 and 3.3.

Observing that $E \xi_{i}(t)=E \eta_{i}(t)=0$,

$$
\begin{aligned}
E \xi_{i}^{2}(t)= & c_{1}^{2}(t)\left\{M(2 t)-M^{2}(t)\right\} \\
E \eta_{i}^{2}(t)= & c_{1}^{2}(t)\left\{M^{\prime \prime}(t)-\left(M^{\prime}(t)\right)^{2}\right\}+2 c_{1}(t) c_{2}(t)\left\{M^{\prime}(2 t)\right. \\
& \left.-M(t) M^{\prime}(t)\right\}+c_{2}^{2}(t)\left\{M(2 t)-M^{2}(t)\right\}
\end{aligned}
$$

and

$$
E \xi_{i}(t) \eta_{i}(t)=c_{1}^{2}(t)\left\{M^{\prime}(2 t)-M(t) M^{\prime}(t)\right\}+c_{1}(t) c_{2}(t)\left\{M(2 t)-M^{2}(t)\right\}
$$

the formulas for $\sigma^{2}$ and $v^{2}$ can be easily derived from Lemmas 3.2 and 3.3 .

Proof of Theorem 1.3. In the light of Lemmas 3.2 and 3.3 it is enough to show that

$$
n^{-1 / \alpha} K(n) \sum_{1 \leqslant i \leqslant n}\left\{X_{i} \exp \left(\tau X_{i}\right)-E X_{i} \exp \left(\tau X_{i}\right)\right\} \stackrel{\mathscr{D}}{\rightarrow} \xi_{\alpha}
$$

and

$$
n^{-1 / \alpha} K(n) \sum_{1 \leqslant i \leqslant n}\left\{\exp \left(\tau X_{i}\right)-M(\tau)\right\} \stackrel{P}{\rightarrow} 0 .
$$

The convergence in distribution in (3.15) follows immediately from the assumption that $X \exp (\tau X)$ is in the domain of attraction of $\xi_{\alpha}$.

Observing that $|X| I\{X \leqslant 0\} \exp (\tau X)$ is a bounded random variable, Theorem 7.7 in Durrett (1991) yields that

$$
P\{X \exp (\tau X)>t\}=t^{-\alpha} K_{1}(t)
$$

where $K_{1}(t)$ is a slowly varying function at $\infty$. For $t$ large enough we get that

$$
\begin{aligned}
P\{\exp (\tau X) \leqslant t\} & =P\left\{X \leqslant \frac{1}{\tau} \log t\right\} \\
& =P\left\{X \exp (\tau x) \leqslant \frac{t}{\tau} \log t\right\}=t^{-\alpha} \tau^{\alpha}(\log t)^{-\alpha} K_{1}\left(\frac{t}{\tau} \log t\right) .
\end{aligned}
$$

Karamata's theorem (cf. Bingham et al. 1987, p. 21) yields that for any $\varepsilon>0$

$$
\frac{K_{1}((t / \tau) \log t)}{K_{1}(t)}=\mathrm{O}\left((\log t)^{\varepsilon}\right) \quad \text { as } t \rightarrow \infty
$$


Using again Theorem 7.7 in Durrett (1991) we can find a slowly varying function $K^{*}(n)$ such that

$$
n^{-1 / \alpha} K^{*}(n) \sum_{1 \leqslant i \leqslant n}\left\{\exp \left(\tau X_{i}\right)-M(\tau)\right\} \stackrel{\mathscr{D}}{\rightarrow} \xi_{\alpha} .
$$

Comparing the tails in (3.17) and (3.18) we get from (3.18) that

$$
\lim _{n \rightarrow \infty} K(n) / K^{*}(n)=0
$$

and therefore the proof of $(3.16)$ is complete.

Proof of Theorem 2.1. Since we can follow the proof of Theorem 1.2 very closely we just give an outline. Elementary arguments similar to those proving Lemma 3.1 give that conditionally on $\boldsymbol{X}_{n}$

$$
\begin{aligned}
& \sup _{0 \leqslant t \leqslant T}\left|M_{m, n}(t)-M_{n}(t)\right| \rightarrow 0 \quad \text { a.s. } \\
& \sup _{0 \leqslant t \leqslant T}\left|M_{m, n}^{\prime}(t)-M_{n}^{\prime}(t)\right| \rightarrow 0 \quad \text { a.s. }
\end{aligned}
$$

and

$$
\sup _{0 \leqslant t \leqslant T}\left|M_{m, n}^{\prime \prime}(t)-M_{n}^{\prime \prime} c(t)\right| \rightarrow 0 \quad \text { a.s. }
$$

as $\min (m, n) \rightarrow \infty$. The consistency of $\hat{\tau}_{m, n}$ and $\hat{c}_{m, n}$ can be derived from (3.19) and (3.20) along the lines of the proof of Theorem 1.1. Using analogues of Lemmas 3.2 and 3.3 what we need is a central limit theorem (conditionally) for sums in the form of

$$
m^{1 / 2}\left\{\frac{1}{m} \sum_{1 \leqslant i \leqslant n}\left(b_{0} \exp \left(\tau X_{i}^{*}\right)+b_{1} X_{i}^{*} \exp \left(\tau X_{i}^{*}\right)\right)-\frac{1}{n} \sum_{1 \leqslant i \leqslant n}\left(b_{0} \exp \left(\tau X_{i}\right)+b_{1}\left(X_{1} \exp \left(\tau X_{i}\right)\right)\right)\right\} .
$$

Since $E X^{2} \exp (2 \tau X)<\infty$, the required central limit theorem follows from Bickel and Freedman (1981).

\section{Acknowledgements}

We are grateful to the referee for pointing out several inaccuracies in the original version of our paper.

\section{References}

Bickel, P.J., Freedman, D.A., 1981. Some asymptotic theory for the bootstrap. Ann. Statist. 9, 1196-1217.

Bingham, N.H., Goldie, C.M., Teugels, J.L., 1987. Regular Variation. Cambridge University Press, Cambridge.

Clark, J.S., 1998. Why trees migrate so fast: Confronting theory with dispersal biology and the paleo record. American Naturalist 152, 204-224.

Csörgö, S., 1980. The empirical moment generating function. In: Gnedenko, B.V., Puri, M.L., Vincze, I. (Eds.), Nonparametric Statistical Inference, Vol. I. North-Holland, Amsterdam, pp. 139-150.

Csörgö, S., Teugels, J.L., 1990. Empirical Laplace transform and approximation of compound distributions. J. Appl. Probab. $27,88-101$. Durrett, R., 1991. Probability: Theory and Examples. Wadsworth, Pacific Grove.

Efron, B., 1979. Bootstrap methods. Another look at the jackknife. Ann. Statist. 7, 1-26.

Hardy, G.H., 1996. A Course of Pure Mathematics, 10th Edition. Cambridge University Press, Cambridge.

Kot, M., Lewis, M.A., van den Driessche, P., 1996. Dispersal data and the spread of invading organisms. Ecology 77, $2027-2042$.

Shao, J., Tu, D., 1995. The Jackknife and Bootstrap. Springer, Berlin.

Weinberger, H.F., 1978. Asymptotic behavior of a model in population genetics. In: Chadam, J.M. (Ed.), Nonlinear Partial Differential Equations and Applications, Lecture Notes in Mathematics, Vol. 648. Springer, Berlin.

Weinberger, H.F., 1982. Long-time behavior of a class of biological models. SIAM J. Math. Anal. 13, 353-396. 